\title{
Lina Stern (1878-1968): Physiologin und Biochemikerin, erste Professorin an der Universität Genf und Opfer stalinistischer Prozesse*
}

\author{
J. J. Dreifuss, N. Tikhonov
}

Lina Stern wurde als Kind einer deutschsprachigen, jüdischen Familie in Libau (heute Liepaya, Lettland) geboren und verbrachte dort ihre Kindheit und Jugend. Libau gehörte damals zum russischen Reich, wo für junge Frauen, die sich einem akademischen Studium zuwenden wollten, kaum Möglichkeiten bestanden. Die kaiserlichen Universitäten nahmen keine Studentinnen auf, und die wenigen Plätze an den Frauenhochschulen waren hauptsächlich für orthodoxe Russinnen bestimmt. Als Lina Stern als Zwanzigjährige in die Schweiz reiste, um wie viele ihrer osteuropäischen Landsleute ein Studium im Ausland zu beginnen, hätte sie niemals gedacht, dass sie mehr als ein Vierteljahrhundert in Genf bleiben würde. Sie schrieb sich in der medizinischen Fakultät ein und entwickelte schnell

\section{Abbildung 1}

Die junge Lina Stern.

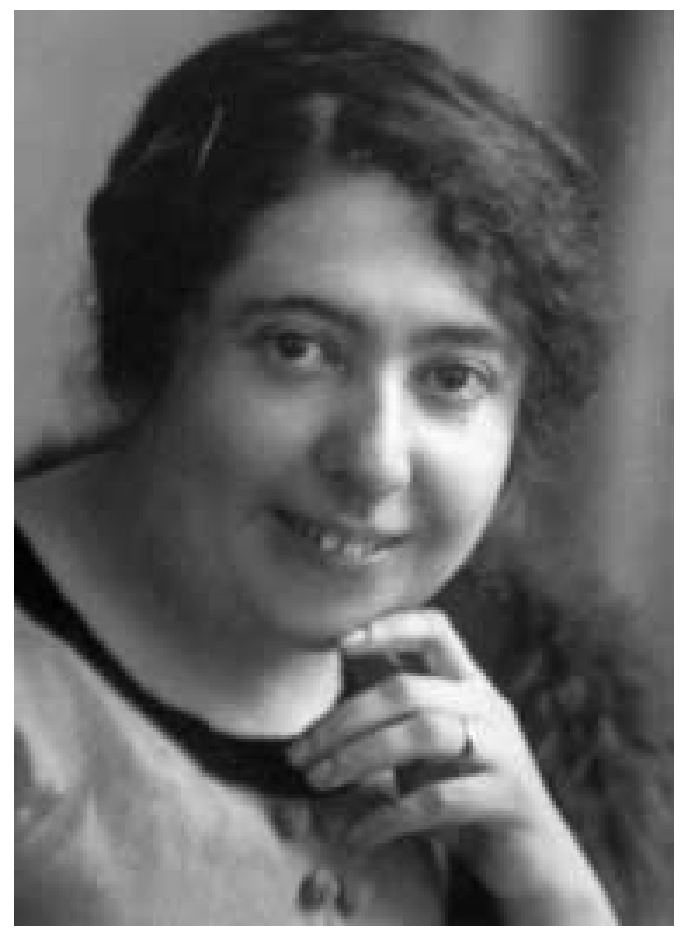

ein besonderes Interesse für die Physiologie. Der Lehrstuhlinhaber dieses Faches, Jean-Louis Prevost der Jüngere (1838-1927), erlaubte Lina Stern, im Laboratorium zu arbeiten, und schon im Jahre 1902 - noch vor Abschluss ihres Studiums - erschien ihr erster Artikel über eine tierexperimentelle Studie. Wiederum war es Prevost, der die Forschungsarbeit betreute, für welche sie 1903 den Doktortitel erhielt. Danach bot er Lina Stern eine Assistentenstelle an, die ihr erlaubte, ihre Forschungsarbeit in Genf weiterzuführen. Ihre akademische Karriere befand sich auf gutem Wege: 1906 wurde sie Privatdozentin und 1918 die erste Frau Professor an der Universität Genf [1].

Zusammen mit Federico Battelli (1867-1941) der 1913 Prevosts Nachfolger wurde - studierte sie die Zellatmung, die Umwandlung eingenommener Nahrungsmittel und eingeatmeten Sauerstoffs in jene Energie, welche die Funktionsfähigkeit der Gewebe und der Organe gewährleistet [2, 3]. Diese und ähnliche Arbeiten führten in den dreissiger Jahren zur Entdeckung des sogenannten Zitronensäurezyklus, in dem verschiedene organische Säuren in den Mitochondrien oxydiert werden. Zwischen 1905 und 1914 erschienen mehr als 30 Arbeiten von Battelli und Stern auf dem Gebiet der Oxydoreduktion, die meisten auf Deutsch in der «Biochemischen Zeitschrift». Sir Hans Krebs zitiert die beiden Genfer Autoren als Vorgänger in der Rede, die er bei der Überreichung des Nobelpreises für Chemie hielt [4].

Vor dem Ersten Weltkrieg studierten viel mehr ausländische als schweizerische Studentinnen und Studenten an der Universität Genf. Kurz nach Kriegsbeginn fiel deren Anzahl jedoch stark zurück, da viele aus wirtschaftlichen Gründen unser Land verlassen mussten. Wahrscheinlich gab die Befürchtung der Genfer Hochschulbehörden, Lina Stern möge im Falle eines günstigen Angebots Genf verlassen, den Ausschlag dafür, dass sie kurz vor Kriegsende zur ausserordentlichen Professorin und Inhaberin des Lehrstuhles für physiologische Chemie an der 
medizinischen Fakultät ernannt wurde. Obwohl mit dieser Beförderung zusätzliche Unterrichtsstunden verbunden waren, erfolgte sie ohne Mehrausgaben für den Staat: ihr Arbeitsplatz blieb in Battellis Institut. Sie hatte als Professorin jedoch jene Unabhängigkeit errungen, die ihr den Einstieg in ein neues Forschungsgebiet erlaubte, und so entschloss sie sich für den Einstieg in neurowissenschaftliche Themen. Ihre Versuche führten sie dazu, die Existenz einer «Blut-Hirn-Schranke» und analoger Übergänge zu postulieren. Als Lina Stern 1921 als erste Frau Mitglied der medizinischen Gesellschaft von Genf wurde, hielt sie über dieses Thema einen Vortrag, der noch im gleichen Jahre in der Zeitschrift erschien, die der berühmte Zürcher Neurologe und russische Adelige Constantin von Monakow (1853-1930) damals herausgab [5].

Wie in Zürich hatte sich auch in Genf zu Beginn des 20. Jahrhunderts eine bedeutende Kolonie von im Exil lebenden oder an der Universität studierenden Russinnen und Russen niedergelassen. Unter ihnen befanden sich zwei Personen, die Lina Stern dazu bewegten, Genf zu verlassen und nach Moskau umzusiedeln [6]. Alexei Bach, ein Mitglied der heimlichen revolutionären Organisation «Der Wille des Volkes», hatte Russland 1889 verlassen und liess sich zuerst in Paris und dann in Genf nieder. Nach der Oktoberrevolution von 1917 kehrte er nach Russland zurück, um in Moskau ein chemisches Forschungsinstitut zu leiten. Der zweite war Boris Zbarski, der in Genf Medizin studiert hatte und nach Lenins Tod im Laboratorium des Leninmausoleums arbeitete. Nachdem Lina Sterns Versuch, ihre ausserordentliche Professur in eine ordentliche umzuwandeln, gescheitert war, konnten diese beiden Russen sie überzeugen, eine Stellung als Institutsleiterin und Hochschulprofessorin in Moskau anzunehmen. So verliess Lina Stern Genf im März 1925. Ihre letzten Genfer Arbeiten betrafen die Entwicklung der Blut-Hirn-Schranke vor und nach der Geburt bei verschiedenen Säugetierarten sowie die BlutPlazenta-Schranke.

Lina Stern benötigte einige Jahre, um sich in einem Lande einzuleben, dessen Sprache sie zwar beherrschte, jedoch mit einem französischen Akzent sprach. Mit eisernem Willen gelang es ihr schliesslich, sich in einem Arbeitsgebiet durchzusetzen, das stark vom bekannten russischen Physiologen Ivan Pavlov und seinen Schülern dominiert war. Von 1929 bis 1948 leitete sie ihr eigenes physiologisches Forschungsinstitut, das zuerst direkt dem Ministerium, später der Akademie der Wissenschaften unterstellt war. Unter ihren zahlreichen Assistenten und wissenschaftlichen Mitarbeitern befanden sich ein hoher Anteil Frauen und einige Ausländer, die sie im Sinne einer internationalen wissenschaftlichen Zusammenarbeit für kürzere oder längere Zeit in ihrem Institut aufnahm. Lina Stern war sehr sprachbegabt; sie reiste öfters und nahm gern an internationalen Kongressen teil. Zwischen 1927 bis 1934 richtete sie mehrere Mitteilungen an die Pariser «Société de Biologie» und gründete in Russland die «Zeitschrift für Biologie und experimentelle Medizin». 1932 wurde sie in die berühmte Deutsche Akademie der Naturforscher Leopoldina aufgenommen und erhielt im selben Jahr beträchtliche Forschungsgelder von der Rockefeller-Stiftung. 1938 trat sie der kommunistischen Partei bei und wurde 1939, wieder auf Veranlassung Alexei Bachs, als erste Frau zum ordentlichen Mitglied der Russischen Akademie der Wissenschaften gewählt [7].

Der Ausbruch des Zweiten Weltkrieges überschattete den weiteren Werdegang von Lina Stern. Der deutsch-russische Pakt ermöglichte Russland, Ostpolen zu besetzen und Finnland anzugreifen. Als im Sommer 1941 die deutsche Armee Russland überfiel und sowohl Leningrad wie Moskau bedrohte, wurden wichtige Persönlichkeiten aufgefordert, diese Städte zu verlassen und sich in sichere Landesteile zurückzuziehen. Wie andere Akademiker zog Lina Stern nach Kasachstan, wo sie versuchte, ihre Forschungsarbeiten weiterzuführen. Die äusseren Umstände erforderten es, dass akutmedizinische Themen in den Vordergrund traten, so die Behandlung von Schockzuständen, Infektionskrankheiten oder Herzstillstand. Die Behörden schätzten Lina Stern in hohem Masse und beriefen sie im Jahre 1942 in den Ausschuss mehrerer neugegründeter «antifaschistischer Komitees», die hauptsächlich in Nordamerika und Grossbritannien für politische und finanzielle Unterstützung werben sollten. 1943 erhielt Lina Stern einen Stalin-Preis für ihre wissenschaftlichen Arbeiten über die Blut-Hirn-Schranke: Sie wurde mit dem Orden der roten Fahne und des roten Sterns ausgezeichnet. Ein Jahr später wurde sie in die neu gegründete Russische Akademie der medizinischen Wissenschaften aufgenommen.

Dank ihrer guten Kontakte ins Ausland verfügte Lina Stern bereits kurz nach Kriegsende über Streptomycin, das erste Antibiotikum, das gegen den Tuberkulosebazillus wirksam war und das die Forschergruppe um Selman Waksman im Jahre 1944 in den USA entdeckt hatte. Mit diesem Medikament konnten etliche russische Kinder, die an tuberkulöser Hirnhautentzündung litten, gerettet werden. 
Alles schien Lina Stern zu gelingen. 1943 wurde sie von einer Person, die sich als Abgeordnete des Gesundheitsministers ausgab, aufgefordert, sich von zwei Mitredaktoren der «Zeitschrift für Biologie und experimentelle Medizin» zu trennen, da der Zentrale Ausschuss der Partei beschlossen hätte, die Zahl der jüdischen Mediziner in leitenden Stellungen stark zu vermindern. Empört sandte sie einen Brief an Stalin, der zu einem Gespräch zwischen Lina Stern und Georgy Malenkov führte. Tatsächlich gelang es ihr, Malenkov zu überzeugen, nichts an der Zusammensetzung der Redaktion zu ändern.

Im Frühjahr 1947 jedoch begann sich der Fall von Lina Stern abzuzeichnen. Es wurde ihr nicht erlaubt, an einem Kongress im Ausland teilzunehmen. Einige Mitglieder der Akademie erhielten den Auftrag, die Produktivität ihres Instituts zu «bewerten» - in Tat und Wahrheit zu kritisieren. 1948 wurde ihr Institut geschlossen unter dem Vorwand des «Kosmopolitismus», Lina Stern habe zu oft Kontakte mit dem Ausland gepflegt. Zudem würden ihre Ideen nicht mit Lyssenkos Lehre übereinstimmen, die Stalin zur Staatsdoktrin erhoben hatte. Trofim Lyssenko verdammte die moderne Genetik als «bürgerliche und rassistische Ideologie» und glaubte an das uralte Konzept der Übertragbarkeit erworbener Merkmale.

Effektiv stellte der Lyssenkismus nur einen der Archaismen dar, die während Stalins Herrschaft die sowjetische Wissenschaftspolitik prägten. $\mathrm{Zu}$ Beginns des «Kalten Krieges» ging es nicht primär um pseudo-wissenschaftliche Meinungsunterschiede, sondern um eine Russifizierung der Wissenschaften und deren Kontrolle durch Partei und Regierung. Das kurioseste Beispiel bildete wohl Stalins Unterstützung von Olga Lepeshinskayas «Widerlegung der Zellehre». Damit wurde eine der grundlegendsten biologischen Theorien der ersten Hälfte des 19. Jahrhunderts verlassen - zugunsten einer Form von «spontaner Generation», wie sie in der Vormoderne diskutiert wurde. Die alte Dame, die in den ersten Jahren des 20. Jahrhunderts zur gleichen Zeit wie Lenin in Genf gelebt hatte, war nur kurz die Zierde der sowjetischen Wissenschaft [8].

Weniger verblüffend dagegen die Anerkennung von Ivan Pavlov, der 1936 im hohen Alter von 87 Jahren als Vater der «bedingten Reflexe» starb und 1904 als erster Physiologe den Nobelpreis für Medizin erhalten hatte. So wie die Lehren Lyssenkos 1948 von der Akademie für Agrarwissenschaften zur Leitidee erklärt und Lyssenko zum Prototyp des «fortschrittlichen sowjetischen Wissenschaftlers» gekrönt wurde, erhoben die Akademien der Wissenschaften und der medizinischen Wissenschaften an einer Sitzung im Jahr 1950 die Lehren Pavlovs zum obligatorischen Vorbild für alle Forscher und Ärzte, die in den Neurowissenschaften oder in der Neuropsychiatrie tätig waren [9]. Was genau die Lehren Pavlovs waren, bestimmten einige linientreue Schüler, die sich als Pavlovs echte geistige Erben betrachteten, hauptsächlich das Akademiemitglied K. M. Bykov. Etliche andere berühmte Schüler Pavlovs wie Orbeli und Beritashvili, die mit ihm noch im Organisationskomitee des 15. Internationalen Kongresses für Physiologie (1935, Leningrad und Moskau) sassen, die aber angeblich auf Abwege gekommen waren, wurden streng getadelt und aufgefordert, sich anzupassen.

Wissenschaftler, die nicht am Modell des bedingten Lernens und dessen Übertragbarkeit arbeiteten, wurden als Söldner der «bürgerlichen, idealistischen Wissenschaft» angezeigt und verklagt. Da Lina Stern angeblich dieser Kategorie angehörte, wurde sie während jener Sitzung 1950 streng angegriffen. Entgegen Orbeli, Beritashvili und anderen war sie nicht im Sitzungssaal anwesend und konnte sich nicht verteidigen. Ihr Institut war ja schon zwei Jahre zuvor geschlossen worden, ihre Mitarbeiter und die wissenschaftlichen Geräte waren inzwischen an andere Institute gelangt - darunter jenes von Bykov in Leningrad. Ende Januar 1949 wurde sie von Beamten der Geheimpolizei verhaftet und ins Lubianka-Gefängnis gebracht, wo politische Gefangene eingesperrt wurden. Drei Jahre und acht Monate musste Lina Stern dort verbringen, und zudem einige unangenehme Zwischenspiele im Lefortovo-Gefängnis erleben, das «speziellen Befragungen» diente. Die Hauptanklagepunkte lauteten auf Spionage, antisowjetische Aktivitäten und Vorbereitung von terroristischen Angriffen.

Seit dem Ende des sowjetischen Regimes, das zur Öffnung der Archive führte, weiss man, dass Lina Stern Mitangeklagte eines geheimen militärischen Prozesses war, der sich gegen vierzehn leitende Mitglieder des Jüdischen Antifaschistischen Komitees richtete. Nach einer dreijährigen Instruktion wurden im Juni 1952 zwölf von ihnen zum Tod verurteilt und im Juli 1952 erschossen. Ein Dreizehnter verstarb im Gefängnis. Einzig Lina Stern überlebte; sie wurde zu dreieinhalb Jahren Haftstrafe verurteilt - also die Zeit ihrer Verhaftung in der Lubianka - gefolgt von fünf Jahren Wohnsitzanweisung in einem abgelegenen Ort in Kasachstan. Ihr Exil in Zentralasien dauerte zum Glück weniger als ein Jahr, denn Mitte 1953, kurz nach dem Tod Stalins, 
Abbildung 2

Lina Stern in den 1950er Jahren. Sammlung Dr. Pierre Rentchnick.

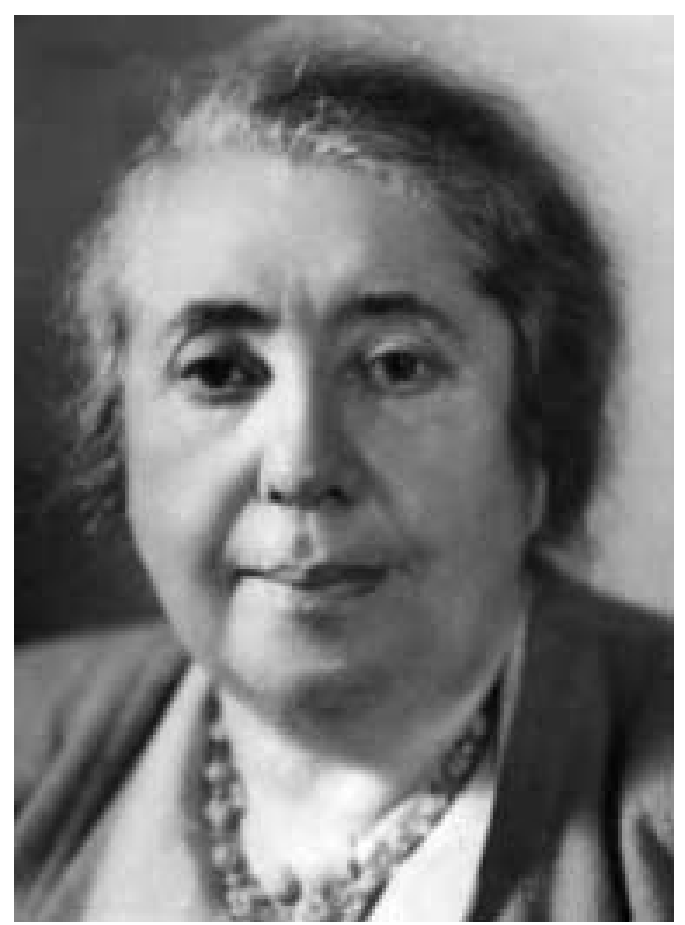

konnte sie nach Moskau zurückkehren, wo sie wieder das Gehalt und die Privilegien eines Akademiemitgliedes erhielt. Die 75jährige forschte nun auf dem Gebiet der biologischen Auswirkungen radioaktiver Strahlung, ein aktuelles Thema im Atomzeitalter. Bereits 1955 wurden die bereits vollzogenen Urteile gegen die mitangeklagten Mitglieder des Ausschusses des Jüdischen Antifaschistischen Komitees aufgehoben, und ihre Familienangehörigen konnten Sibirien verlassen [10].

Dr. Leon Weber-Bauler, der während des Zweiten Weltkriegs die in Genf erscheinende medizinische Wochenschrift «Médecine et Hygiène» gründete, hatte zuvor lange in Russland gelebt. Er veröffentliche regelmässig Kurzartikel zu medizinischen Themen in der Sowjetunion. Lina Sterns ehemalige Kollegen und Freunde in Genf waren deshalb mehr oder weniger über ihre Schwierigkeiten informiert, man vermutete sogar das Schlimmste. Die Erleichterung war gross, als man erfuhr, dass sie dem Tod entgangen war. Nach ihrer Rückkehr nach Moskau korrespondierte Lina Stern über Jahre mit dem Genfer Rechtsprofessor Maurice Battelli, dem Sohn ihres ehemaligen, in der Zwischenzeit verstorbenen Kollegen. Der Nachfolger von Weber-Bauler als Herausgeber von «Médecine et
Hygiène», Dr. Pierre Rentchnick, versuchte vergebens, Lina Stern in Moskau oder anderswo zu treffen, als er 1954 eine längere Reise durch Russland unternahm. Dafür traf er sie 1956 und 1962 und erfuhr einiges über ihre schwierigen Jahre.

Erst 1958, im Jahre ihres 80. Geburtstags also, wurde Lina Stern offiziell rehabilitiert. Diese beiden Ereignisse wurden in einem grossen Hörsaal der Akademie mit reichem Blumenschmuck gefeiert. Lina Stern leitete ihre Dankesrede humorvoll mit den folgenden Worten ein: «Freunde und Zuhörer, ich hab' den Eindruck, an einer allgemeinen Wiederholung meiner Bestattung teilzunehmen.»

1960 erhielt Lina Stern den Titel eines Doktors honoris causa der Universität Genf, die damit ihre erste Professorin ehrte. Die Laudatio hielt fest, sie habe durch ihre Forschungsarbeiten auf den Gebieten der Biochemie und der Neurowissenschaften in hohem Masse «zur Bedeutung der Universität von Genf beigetragen». Lina Stern verfasste noch weitere zehn Artikel bis zu ihrem Todesjahr 1968.

\section{Literatur}

1 Posternak J. La physiologie à la Faculté de médecine de Genève. In: Cramer $\mathrm{M}$, Starobinski J (dir.). Centenaire de la Faculté de médecine de l'Université de Genève. Genève: Médecine et Hygiène; 1978.

2 Battelli F, Stern L. Die Oxydationsfermente. Ergeb Physiol 1912;12:96-268.

3 Stern L. Über den Mechanismus der Oxydationsvorgänge im Tierorganismus. Berl Klin Wochenschr 1914;22:1028-34.

4 Krebs HA. The citric acid cycle. Nobel lecture, December 11, 1953, Stockholm.

5 Stern L. Le liquide céphalorachidien au point de vue de ses rapports avec la circulation sanguine et avec les éléments de l'axe cérébro-spinal. Schweiz Archiv Neurol Psychiatr 1921;8:215-32.

6 Klein E. Lina S. Stern. In: Klein E. Führende Frauen Europas. München: Ernst Reinhardt; 1930.

7 Malkin V, Rosin I. Lina Solomonovna Stern. Moskau: Nauka; 1987.

8 Rapoport I. The doctor's plots of 1953. Cambridge: Harvard University Press; 1991.

9 Academy of Sciences of the USSR. Scientific Session of the Teachings of Academician I. P. Pavlov. Moscow: Foreign Languages Publishing House; 1951.

10 Rubenstein J, Naumov VP (eds.). Stalin's Secret Pogrom. The Postwar Inquisition of the Jewish Antifascist Committee. New Haven: Yale University Press; 2001. 